\title{
A simple electrical stimulation cell cullture system on the myelination of dorsal root ganglia and Schwann cells
}

\author{
Zhuowen Liang ${ }^{1}$, Tao Leï ${ }^{2}$, Shuang Wang ${ }^{3}$, ZhuoJing Luo' \& XueYu Hu*,1
}

\begin{abstract}
A large number of animal experiments and clinical trials have confirmed that electrical stimulation can accelerate the growth of axons and recovery of motor function, all of which are inseparable from the formation of myelin. Therefore, establishment of a suitable electrical stimulation platform to study the effects of electrical stimulation on the myelin process of dorsal root ganglia and Schwann cells is of great significance for understanding the recovery of electrical stimulation. We designed a simple conductive glass cell culture system to overcome the shortcomings of direct contact of the electrode with the culture solution, and the number of culture chambers can be selected based on the purpose of the experiment in order to reduce experimental time and cost.
\end{abstract}

\section{METHOD SUMMARY}

Here, we propose a new electrical stimulation cell culture system for exposing neurons/ Schwann cells to electrical stimulation to induce cell differentiation, migration and myelination. The device is simple, convenient and can be used to study the electrical stimulation effect and electrophysiological characteristics of nerve cells in vitro.

\section{INTRODUCTION}

The development of neurons mainly involves the differentiation of stem cells into neurons, migration and differentiation into mature functional neural circuits. The three processes occur in turn and interact with each other, and electrical signals play a decisive role in the whole process [1]. The nervous system maintains normal physiological functions through spontaneous discharge. When the nervous system is damaged, this balance breaks down, and intracellular $\mathrm{Ca}^{2+}$ overload in neurons accelerates neuronal apoptosis. Intervening in this process by applying electrical stimulation can alleviate or reverse the damage [2-5]. The process, especially in the repair of peripheral nerve injury, is effective. Electrical stimulation is given to patients with median nerve injury caused by human carpal tunnel syndrome. It has been confirmed that short-term, low-frequency electrical stimulation can significantly promote nerve regeneration and stimulate the feeling of innervation and motor function $[6,7]$, however the specific mechanism is not clear. Therefore, the establishment of an in vitro dorsal root ganglion (DRG)Schwann cell (SC)-electric stimulation model provides a basis for further study of the mechanism of electrical stimulation of peripheral nerve myelination.

The entire electro-stimulation culture system has simple assembly, high transparency, sliding disassembly slides, and observation of various imaging devices without transferring and destroying cells.

\section{MATERIALS \& METHODS}

Electrical stimulation apparatus

Electrical stimulation apparatus consisted of a function generator (Agilent Technologies, USA) and an electrical stimulation chamber (homemade) (Figure 1A). The opencircuit voltage waveform was a rectangular wave with amplitude at $6 \mathrm{Vp}$-p and frequency at $10 \mathrm{~Hz}$ (Figure 1B). The electrical stimulation chamber consisted of five parts from top to bottom, including a polystyrene cover (Biologix, China), a conventional hollow polystyrene chamber (Biologix), a biocompatible gasket (Biologix), a conductive glass $(58 \mathrm{~mm} \times 25 \mathrm{~mm} \times 1 \mathrm{~mm}$, length $\times$ width $\times$ thickness, GuLuo Company, China) and a polystyrene fixture (Biologix) (Figure 1A). The hollow polystyrene chamber was surrounded by the biocompatible gasket, and the conductive glass was under the hollow polystyrene chamber. The polystyrene fixture fixed the hollow polystyrene chamber and conductive glass to prevent the exudation of culture medium. The electrical stimulation chamber can be performed in series or parallel circuits according to the experimental design. In our study, three single-chambers were connected in parallel with copper wire and conductive clips (Figure 2A).

\section{Dorsal root ganglion culture}

DRG was collected from newborn 1-day-old Sprague-Dawley (SD) rats, cut into $0.5 \mathrm{ml}$ collagenase and $1 \mathrm{ml} 0.25 \%$ trypsin (without EDTA) and $1 \mathrm{ml} \mathrm{DME/F12} \mathrm{(HyClone,} \mathrm{USA)} \mathrm{for}$ $1 \mathrm{~h}$, and filtered with a $40 \mu \mathrm{m}$ microporous filter to terminate digestion. This was centrifuged at $500 \mathrm{~g} / \mathrm{min}$ for $5 \mathrm{~min}$. Then, the cells were suspended in DME/F12 medium containing 10\% FBS serum (Gibco, USA) at a density of $3 \times 10^{5}$ cells in a conductive glass. After $24 \mathrm{~h}$ in culture, replace the 


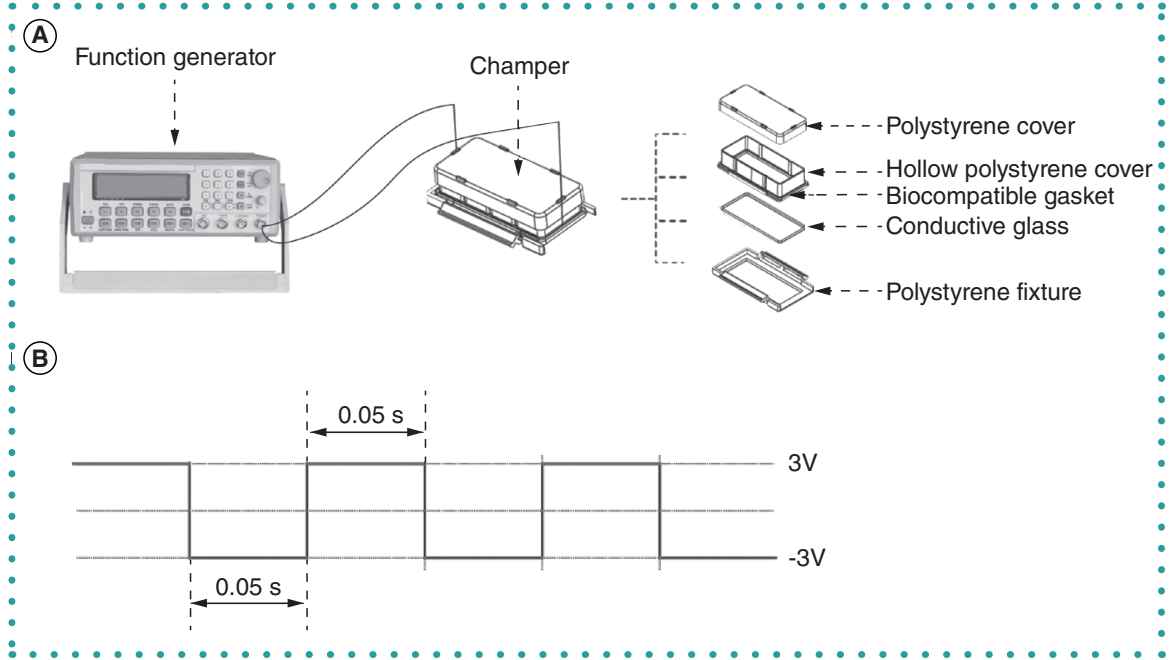

Figure 1. Representation of the electrical stimulation apparatus. (A) The apparatus consists of two parts: a function generator and an electrical stimulation chamber. (B) The open-circuit voltage waveform of the function generator was a rectangular wave with an amplitude of $6 \mathrm{Vp}-\mathrm{p}$ and frequency of $10 \mathrm{~Hz}$.

(A)

(B)
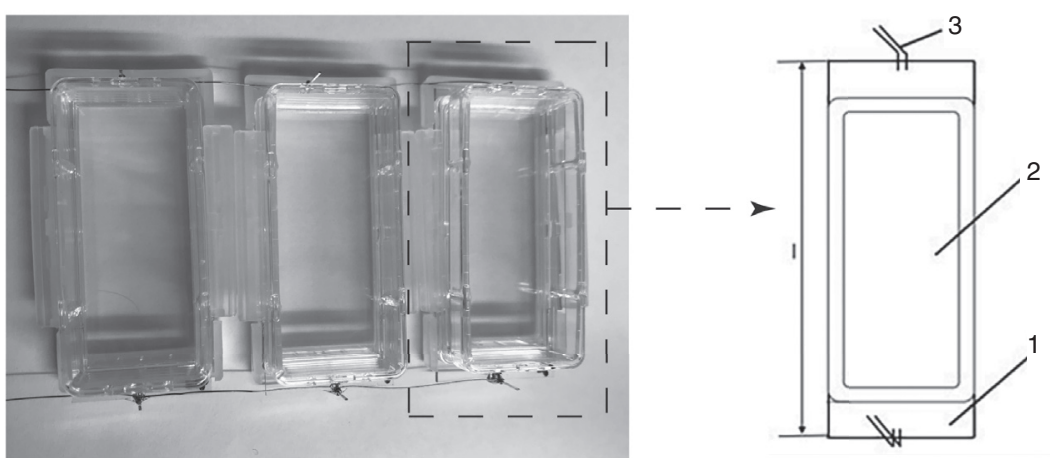

Figure 2. Electrical stimulation chamber. (A) Three single-chambers were connected in parallel with copper wire and conductive clips. (B) Every single-chamber mainly included: 1: conductive glass; 2: cell culture chamber; 3 : conductive clip.

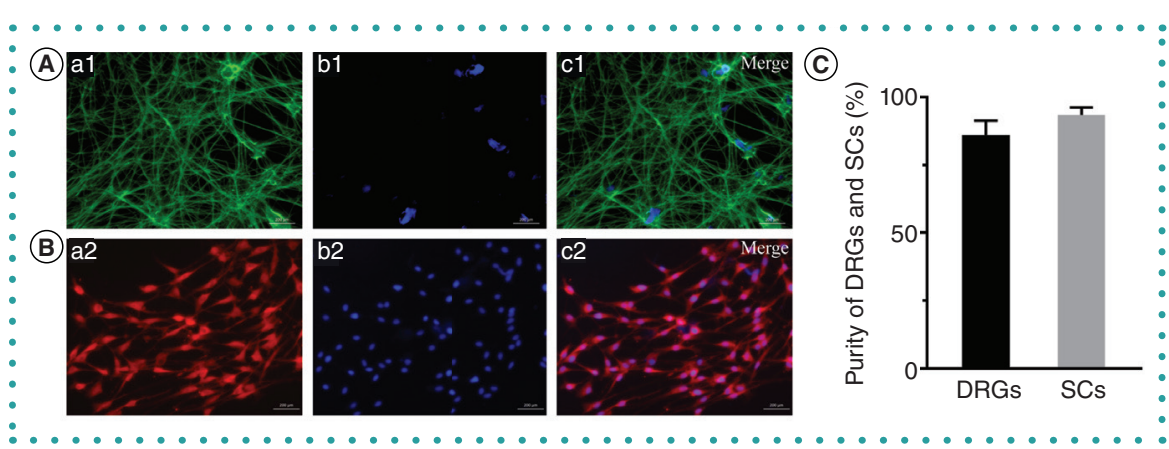

Figure 3. Identification the purity of DRG and SC cells. (A) DRG cells: a1: Immunofluorescence images of axon with marker $\beta$-tubulin III (green); b1: Nuclei were stained with DAPI (blue); c1: Representative image of merged purified DRG neurons. (B) SC cells: a2: Immunofluorescence images of SC with marker S100 (red); b2: Nuclei were stained with DAPI (blue); c2: Representative image of a culture of purified SC. (C) The semi-quantitative results of the purity of DRG and SC cells. DRG: Dorsal root ganglion; SC: Schwann cell.

medium with Neurobasal (Gibco) + B27 (Gibco) + NGF (50 ng/ml) (R\&D Systems, USA)+5-FU (31 $\mu \mathrm{mol} / \mathrm{ml})$ (HSRVEY, USA) for
$48 \mathrm{~h}$ to remove non-DRG cells, and culture was continued for 3 days in medium without 5-FU for experimental use [8].

\section{Schwann cell culture}

The collection method of SCs was adopted from a previously published paper. Bilateral sciatic nerves collected from newborn 1-day-old SD rats were cut into a $5 \mathrm{ml} \mathrm{EP}$ tube, and $1 \mathrm{ml}$ collagenase, $1 \mathrm{ml} 0.25 \%$ trypsin (without EDTA) and $1 \mathrm{ml} \mathrm{DME/F12}$ were added to the EP tube for digestion. This was digested for $30 \mathrm{~min}$, and centrifuged at $500 \mathrm{~g} / \mathrm{min}$ for $5 \mathrm{~min}$. The cells were suspended in DME/F12 medium containing $10 \%$ FBS serum in a $10-\mathrm{cm}$ culture dish at $37^{\circ} \mathrm{C}$ for $24 \mathrm{~h}$, and then cultured with DME/ F12 containing $10 \%$ FBS serum, 5 -FU $(31 \mu \mathrm{mol} / \mathrm{ml})$ and Forskolin $(10 \mu \mathrm{mol} / \mathrm{ml})$ (MCE, USA) for $48 \mathrm{~h}$ for purification. Then, the cells were cultured with DME/F12 medium containing Forskolin $(10 \mu \mathrm{mol} / \mathrm{ml})$ and $10 \%$ FBS serum for $48 \mathrm{~h}$ for experimental use [8].

\section{The identification of purity of DRG \& SC cells}

Ensuring the purity of DRG and SC cells is essential for electrical stimulation of co-culture, so we identified the purity of the DRG and SC cells. The purity of DRG cells was identified by $\beta$-tubulin III antibody (Abcam, USA), the purity of SC cells was identified by $\mathrm{S} 100$ antibody (Abcam), and all the cell counts were identified by nuclear dye DAPI (BIOSS, China). Five visual fields were randomly selected by ImageJ software. The nucleus (DAPI) and cell bodies ( $\beta$-tubulin III and S100) were respectively counted. The cell purity was calculated by the ratio of cell bodies to the nucleus.

\section{$\mathrm{DRG} / \mathrm{SC}$ co-culture}

The SC were digested with $0.25 \%$ trypsin (without EDTA) for $5 \mathrm{~min}$, then centrifuged at $500 \mathrm{~g} / \mathrm{min}$ for $5 \mathrm{~min}$, then resuspended in DME/F12 medium containing 10\% FBS serum. SC were inoculated in a DRG culture plate at a density of $2 \times 10^{5}$ cells/conductive glass for $24 \mathrm{~h}$. Then, the cells were cultured with Neurobasal + B27 + NGF $(50 \mathrm{ng} / \mathrm{ml})$ $+5 \%$ FBS culture medium [8].

\section{Stimulation protocol}

The electrical stimulation chamber underwent ultrasonic cleaning, washing, drying and then disinfecting by ultraviolet irradiation (12 h) before use. After disinfection, the surface of the conductive glass was dried with dextran polylysine for $3 \mathrm{~h}$. 
DRG/SC were divided into a control (Ctrl) group and an electrical stimulation (ES) group after $24 \mathrm{~h}$ of co-culture. The ES group was given an electrical stimulation with rectangular wave, $6 \mathrm{Vpp}, 10 \mathrm{~Hz}, 1 \mathrm{~h} / \mathrm{d}, 7$ days. The Ctrl group had no electrical stimulation. The culture medium in the Ctrl and ES groups were changed every 2 days. During the whole process, the electrodes were not in contact with the culture medium and the cells, and the conductivity of the conductive glass was normal.

\section{CCK8 detection}

The aim of CCK8 detection was to test whether the conductive glass culture system was toxic to the cells. When the DRG/SC were cultured for 7 days, the cells in the ES group and the Ctrl group were transferred to a 96-well plate, $100 \mu \mathrm{l}$ medium and $10 \mu \mathrm{l} \mathrm{ССК8}$ solution was added to each well, and incubated for 1-4 $\mathrm{h}$, and the absorbance at $450 \mathrm{~nm}$ was measured with an enzymelabeled instrument.

\section{Immunofluorescence}

The myelination of DRG/SC cells was indicated by the immunofluorescence of myelin basic protein (MBP) protein and myelin protein zero protein (P0). At day 7 , the DRG/SC cells were fixed with $4 \%$ paraformaldehyde for $30 \mathrm{~min}$ at room temperature. Anti-hypo-phosphorylated neurofilament $\mathrm{H}$ (NF200) (Abcam), anti-MBP (BIOSS) and anti-myelin protein zero protein ( $\mathrm{PO}$ ) (Abcam) were incubated overnight at $4^{\circ} \mathrm{C}$ and washed three times with PBS. Secondary antibody including sheep anti-mouse IgG-488 (1:600) (Jackson ImmunoResearch, USA) and donkey anti-rabbit IgG-Cy3 (1:600) (Jackson ImmunoResearch) were added in the dark and incubated for $1 \mathrm{~h}$ at room temperature. After washing three times with PBS, the cells were observed under a Lycra inverted fluorescence microscope imaging system (Carl Zeiss, Germany). Five visual fields were randomly selected. The total intensity of fluorescence and area were analyzed by ImageJ software. The fluorescence density was obtained from the total fluorescence intensity divided by the area.

\section{Western blot}

The expression of MBP protein in DRG/SC was analyzed by western blot. The DRG/SC in the Ctrl group and the ES group were co-cultured for 7 days, lysed with RIPA lysate, and the cell lysate protein concentration was determined by Bradford assay. The total proteins were mixed with $5 \times$ loading buffer (loading buffer: supernatant, 1:4, v:v) and loaded onto a $12 \%$ sodium dodecyl sulfate (SDS) polyacrylamide gel. Electrophoresis was performed to transfer separated protein onto a polyvinylidene fluoride membrane (PVDF, Thermo Fisher Scientific, USA). The membrane was incubated in primary antibody $\operatorname{MBP}(1: 500)$ overnight at $4^{\circ} \mathrm{C}$, and then incubated in secondary antibody (conjugated HRP goat anti-rabbit 1:3000) at room temperature for 1-2 h. Blots were

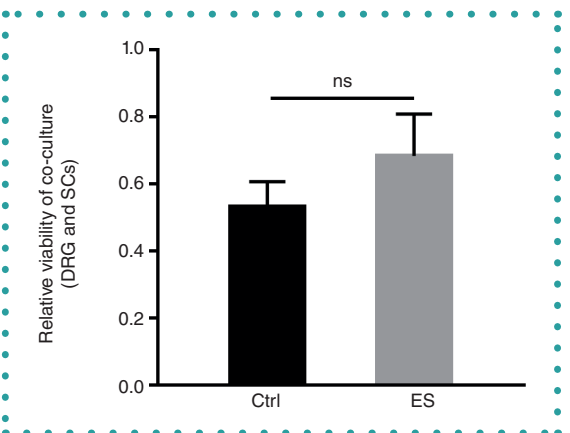

Figure 4. Effects of 7-day electrical stimulation on cell cytotoxicity of DRG/SCs by CCK8. Values represent mean \pm SEM of $3 /$ group, ns indicates $p>0.05$, statistically insignificant compared with the Ctrl group. Ctrl: Control; DRG: Dorsal root ganglion; ES: Electrical stimulation; SC: Schwann cell.

(A)

Control - no electrical stimulation, 7th day
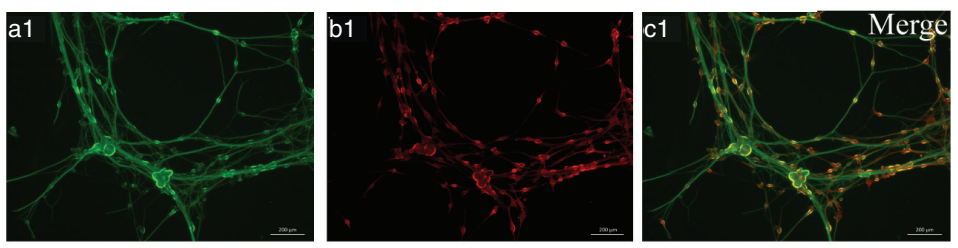

ES - exposure to $6 \mathrm{Vp}-\mathrm{p} 10 \mathrm{~Hz} 1 \mathrm{~h} / \mathrm{d}$, 7th day
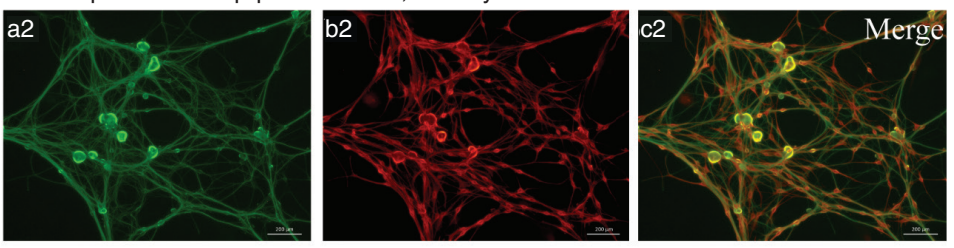

(B)

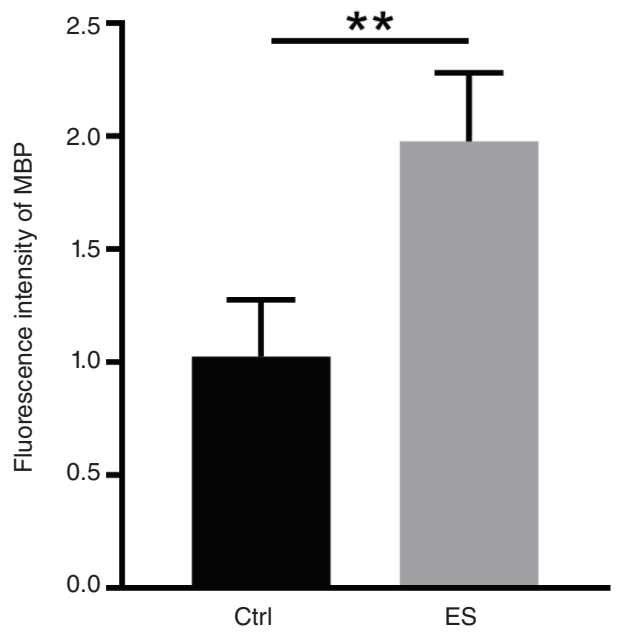

Figure 5. Immunofluorescent for MBP of co-cultured DRG/SC cells after 7-day electrical stimulation. (A) Ctrl group, no electrical stimulation. a1: The neurite network by NF200 staining (green); b1: myelinated segments (red) by MBP staining; $\mathrm{cl}$ : MBP protein is expressed on axons after co-culture 7 days; ES group, exposure to 6 Vp-p,10 Hz. a2: NF200 (green); b2: MBP (red); c2: MBP positive section are abundant throughout the co-cultures after electrical stimulation compared with Ctrl group. (B) The semi-quantitative results of fluorescence intensity of MBP in Ctrl and ES groups. Values represent mean \pm SEM of $3 /$ group, ${ }^{* *} p<0.01$, statistically significant compared with the Ctrl group. Ctrl: Control; DRG: Dorsal root ganglion; ES: Electrical stimulation; MBP: Myelin basic protein; SC: Schwann cell. 
(A) Control - no electrical stimulation, 7th day
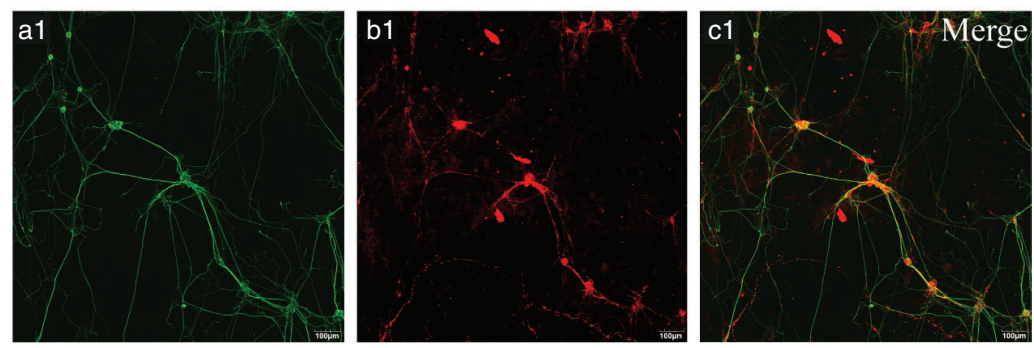

ES - exposure to $6 \mathrm{Vp}-\mathrm{p} 10 \mathrm{~Hz} 1 \mathrm{~h} / \mathrm{d}$, 7th day
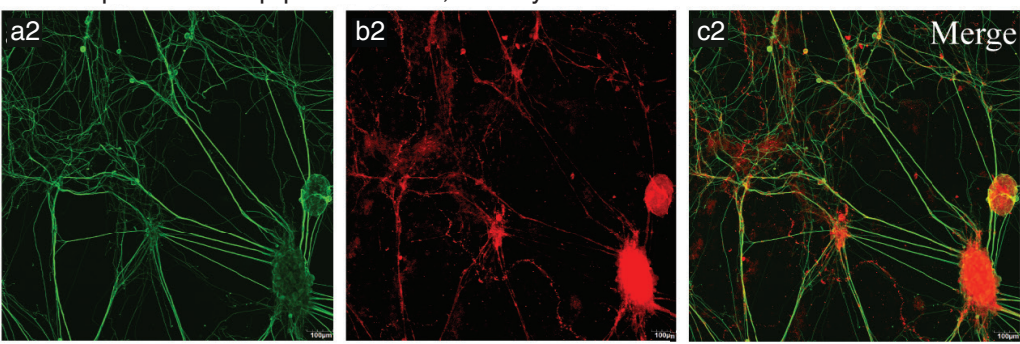

(B)

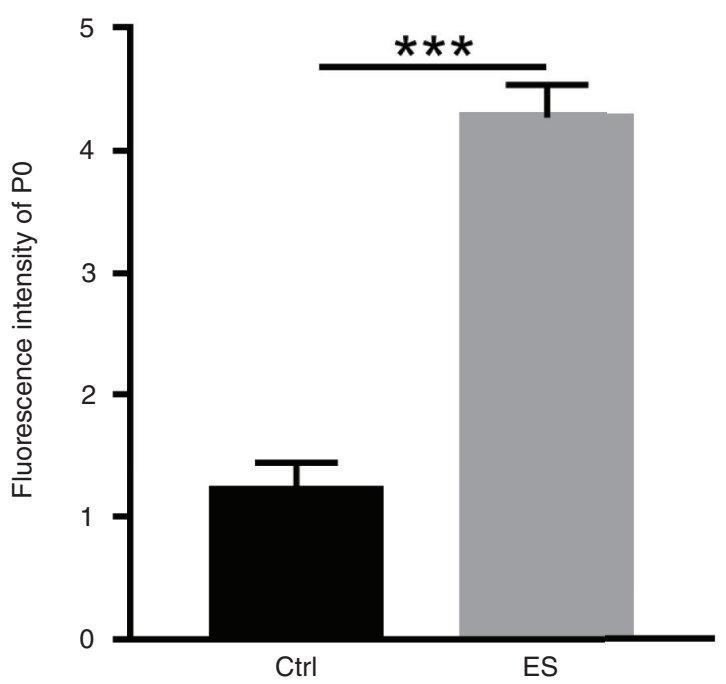

Figure 6. Immunofluorescent for PO of co-cultured DRG/SC cells after 7-day electrical stimulation. (A) Ctrl group, no electrical stimulation. a1: The neurite network by NF200 staining (green); b1: myelinated segments (red) by $\mathrm{P} 0$ staining; $\mathrm{cl}$ : $\mathrm{P} 0$ protein is expressed on axons after co-culture 7 days; ES group, exposure to 6 Vp-p,10 Hz. a2: NF200 (green); b2: P0 (red); c2: P0 positive section are abundant throughout the co-cultures after electrical stimulation compared with Ctrl group. (B) The semi-quantitative results of fluorescence intensity of PO in Ctrl and ES groups. Values represent mean \pm SEM of 3/group, ${ }^{* * *} p<0.001$, statistically significant compared to the Ctrl group. Ctrl: Control; DRG: Dorsal root ganglion; ES: Electrical stimulation; SC: Schwann cell.

developed using GE Amersham Image 600 station, and the bolts were analyzed using Gel-Proanalyzer analysis software, the ratio of the gray value of the target protein band to the gray value of the $\beta$-actin band of the internal reference is the relative expression of the protein.

\section{Statistical analysis}

Results were reported as mean \pm SEM and $p<0.05$ was defined as the threshold of significance. Data were analyzed with the SPSS v 20.0.0 statistical software (IBM, USA). Each in vitro experiment contained a minimum of three replicates. Data presented are representative of at least three experiments. Independent sample t-test analysis was used for comparison between groups.

\section{RESULTS \& DISCUSSION}

The ratio of the counts of DRG neurons ( $\beta$-tubulin III staining) and the counts of nuclei (DAPI staining), indicate the purity of DRG. The high counts ratio of DRG neurons and nuclei $(86 \pm 4.32 \%)$ in Figure 3 C indicated that there were few non-DRG cells, which meant the DRG cells had high purity and were suitable for the co-culture. Similarly, the purity of SC cells was indicated by the ratio of SC maker (S100) and nuclei (DAPI). The high counts ratio of SC and nuclei $(93 \pm 3.99 \%)$ in Figure $3 \mathrm{C}$ indicated that there were few non-SC cells, which meant the SC cells had high purity and were also suitable for the co-culture.

CCK8 cell proliferation-toxicity test showed (Figure 4) that the OD value in the ES group was slightly higher than that in the Ctrl group. However, there was no statistical significance between the two groups. Therefore, it was confirmed that the conductive glass culture system was not toxic to nerve cells.

The immunofluorescence of the MBP and P0 protein of co-cultured DRG/SC cells after 7-day electrical stimulation are shown in Figures 5 \& 6 . The neurite network is indicated by NF200 staining (green), and compact myelinated segments (red) were respectively indicated by $\mathrm{MBP}$ and $\mathrm{PO}$ staining. Protein expression levels of MBP and $\mathrm{PO}$ by immunofluorescence showed that the fluorescence intensity of MBP and $P 0$ in the ES group was significantly higher than those in the Ctrl group $(p<0.01)$. At the same time, western blot showed that the expression of MBP in the ES group was significantly higher than that in the Ctrl group $(p<0.01)$ (Figure 7 ).

The above results confirmed that the conductive glass culture system is safe and non-toxic to the nerve cells and the continuous 7-day electrical stimulation can significantly increase the myelination rate.

Peripheral nerve injury has limited selfhealing ability, resulting in corresponding atrophy of muscle tissue and loss of function. Electrical stimulation can increase the speed and success rate of nerve repair by directly promoting axon growth. At the same 
time, electrical stimulation can increase the activity of SCs and the secretion of neurotrophic factors, but the specific molecular mechanism is still unclear [9-13]. Establishing a simple, effective and reproducible electrostimulation cell culture system is particularly important for studying the interaction mechanism between nerve cells and currents, and for formulating effective clinical electrical stimulation therapy parameters. Compared with the previously reported electrical stimulation equipment [14-19], the electrical stimulation culture system in this study is easy to assemble, the electrode is not in contact with the culture solution and the cells, and it does not produce toxicity. In addition, the current of the system is uniform, and high-definition conductive glass can fulfill various imaging requirements. Moreover, different kinds of experimental demands could be satisfied by using a series or parallel electrical stimulation chambers.

In summary, the electrical stimulation culture system in our study is easy to assemble, simple to operate, inexpensive and can be used for a variety of different experimental applications. The system has a significant stimulative effect on the co-culture of nerve cells into myelin sheath, which provides a good experimental platform for studying the relationship between nerves and electrical signals.

\section{FUTURE PERSPECTIVE}

Electrical stimulation therapy has been widely applied in the clinical disease therapy, especially in CNS diseases (Alzheimer's disease, epilepsy, depression, etc.) and peripheral nerve injury. Electrical stimulation does not only promote the repair of injured nerves but also regulates the abnormal discharge of neurons (epilepsy); however, the specific mechanism has not been fully elucidated. Therefore, a better understanding of the neurophysiological mechanism is of great significance for the choice of treatment method and treatment parameters. Here we designed an electrical stimulation cell culture system based on conductive glass and a conventional hollow polystyrene chamber, which is used to study the electrophysiological mechanism of nerve cells in vitro. The whole electric stimulation culture system is assembled, the material is easy to prepare, the cost is low,
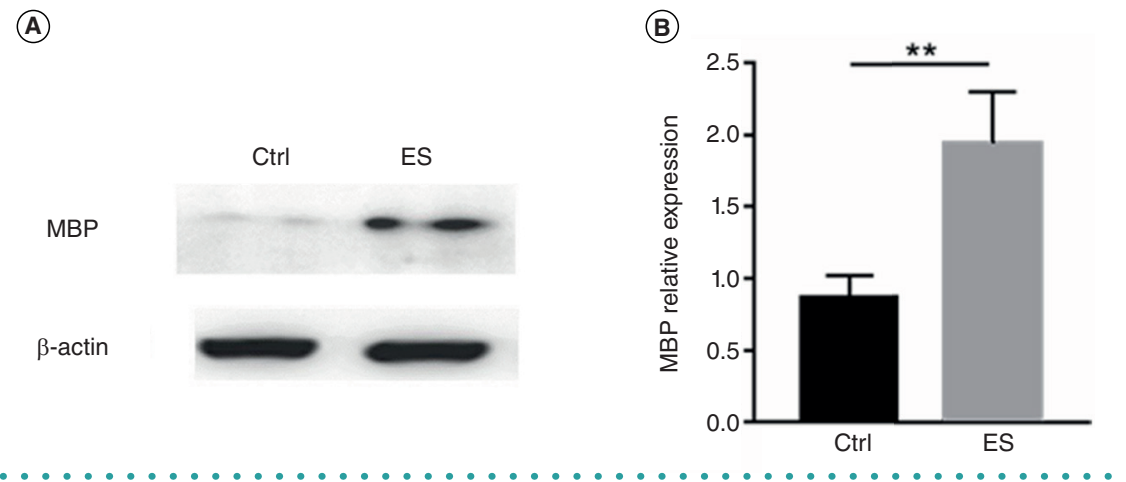

Figure 7. Effects of 7-day electrical stimulation on MBP protein expression of DRG/Scs by western blot. (A) Representative bolt images for MBP protein. (B) Statistical comparisons of MBP protein expression levels in Ctrl and ES groups. Values represent mean \pm SEM of $3 /$ group, ${ }^{* *} p<0.01$, statistically significant compared with the Ctrl group. Ctrl: Control; ES: Electrical stimulation; MBP: Myelin basic protein.

and the hollow polystyrene chamber of culture can be adjusted according to experimental needs. Different kinds of experimental demands could be satisfied by adjusting the connection methods of the electrical stimulation chambers. In addition, the culture system conveniently conforms to requirements of current dominant imaging devices. In the future, the electric stimulation culture system can be used to 3D culture, simulate a more complex culture environment (such as blood or plasma media) and dynamically observe how electrical stimulation affects the interaction between nerve cells in a two-photon imaging system, and further discover the electrophysiological mechanism that electrical stimulation affects nerves cells. We believe that this electrostimulation cell culture system can also be applied to other cellular research to investigate the mechanism of the effects of electronic features on biological tissues.

\section{AUTHOR CONTRIBUTIONS}

ZL and SW designed and performed the experiments, TL, $\mathrm{XH}$ and $\mathrm{ZL}$ analyzed data. $S M$ designed and performed the experiments. ZL and TL co-wrote the paper.

\section{FINANCIAL \& COMPETING INTEREST DISCLOSURE}

The authors acknowledge support from the National Natural Science Foundation of China (grant no. 81572151 and 51577188) and support from the Science and Technology Program in Social Development of Shaanxi Province (2017SF-021). The authors have no other relevant affiliations or financial involvement with any organization or entity with a financial interest in or financial conflict with the subject matter or materials discussed in the manuscript apart from those disclosed.

No writing assistance was utilized in the production of this manuscript.

\section{OPEN ACCESS}

This work is licensed under the AttributionNonCommercial-NoDerivatives 4.0 Unported License. To view a copy of this license, visit http://creativecommons.org/licenses/ by-nc-nd/4.0/

\section{REFERENCES}

1. Stiles J, Jernigan TL. The basics of brain development. Neuropsychol. Rev. 20(4), 327-348 (2010).

2. Egorov AV, Draguhn A. Development of coherent neuronal activity patterns in mammalian cortical networks: Dev. 130(6-8), 412-423 (2013).

3. Kilb W, Kirischuk S, Luhmann HJ. Electrical activity patterns and the functional maturation of the neocortex. Eur. J. Neurosci. 34(10), 1677-1686 (2011).

4. Nave KA, Werner HB. Myelination of the nervous Biol. 30, 503-533 (2014).

5. Spitzer NC. Electrical activity in early neuronal development. Nature 444(7120), 707-712 (2006).

6. Gordon T. Electrical stimulation to enhance axon regeneration after peripheral nerve injuries in animal models and humans. Neurotherapeutics 13(2), 295-310 (2016).

7. Gordon T, Amirjani N, Edwards DC, Chan KM. Brief post-surgical electrical stimulation accelerates axon regeneration and muscle reinnervation without affecting the functional measures in carpal tunnel syndrome patients. Exp. Neurol. 223(1), 192-202 (2010).

8. Maurel P. Preparation of neonatal rat Schwann cells and embryonic dorsal root ganglia neurons for in vitro myelination Studies. Methods Mol. Biol. 1739, 17-37 (2018).

9. Willand MP, Nguyen MA, Borschel GH, Gordon T. Electrical stimulation to promote peripheral nerve regeneration. Neurorehabil Neural Repair 30(5), 490-496 (2016).

10. Gordon T. Nerve regeneration in the peripheral and central nervous systems. J. Physiol. 594(13), 3517-3520 common principles and local hetereogeneity. Mech. system: mechanisms and functions. Annu. Rev. Cell Dev. 
(2016).

11. Alrashdan MS, Sung MA, Kwon YK, Chung HJ, Kim SJ, Lee JH. Effects of combining electrical stimulation with BDNF gene transfer on the regeneration of crushed rat sciatic nerve. Acta Neurochir (Wien) 153(10), 2021-2029 (2011).

12. Koppes AN, Seggio AM, Thompson DM. Neurite outgrowth is significantly increased by the simultaneous presentation of Schwann cells and moderate exogenous electric fields. J. Neural. Eng. 8(4), 046023 (2011).

13. Huang J, Ye Z, Hu X, Lu L, Luo Z. Electrical stimulation induces calcium-dependent release of NGF from cultured Schwann cells. Glia 58(5), 622-631 (2010).
14. Dauben TJ, Ziebart J, Bender T, Zaatreh S, Kreikemeyer B, Bader R. A novel in vitro system for comparative analyses of bone cells and bacteria under electrica stimulation. Biomed. Res. Int. 2016, 5178640 (2016).

15. Mobini S, Leppik L, Barker JH. Direct current electrical stimulation chamber for treating cells in vitro. Biotechniques 60(2), 95-98 (2016).

16. Lozano R, Gilmore KJ, Thompson BC et al. Electrical stimulation enhances the acetylcholine receptors available for neuromuscular junction formation. Acta Biomater. 45, 328-339 (2016).

17. Du Z, Bondarenko O, Wang D, Rouabhia M, Zhang Z. Ex vivo assay of electrical stimulation to rat sciatic nerves: cell behaviors and growth factor expression. J. Cell Physiol. 231(6), 1301-1312 (2016).

18. Lee HU, Blasiak A, Agrawal DR et al. Subcellular electrical stimulation of neurons enhances the myelination of axons by oligodendrocytes. PLOS One 12(7), e0179642 (2017).

19. Ishibashi T, Dakin KA, Stevens B et al. Astrocytes promote myelination in response to electrical impulses. Neuron 49(6), 823-832 (2006). 